necessary. In 21/82 cases INSURE was not successful. In the unsuccessful group patients were not significantly younger and smaller. Procalcitonin levels at the age of one day were significantly higher the group of unsuccessful cases. III-IV Gr. IVH occurred in 6/82 necrotizing enterocolitis in 7/82 and bronchopulmonary dysplasia in $7 / 82$ cases. Complications were more frequent in those cases whose INSURE therapy was unsuccessful.

Conclusions The introduction of INSURE-therapy grossly decreased the need for invasive respiratory support. High procalcitonin levels and clinical manifestations of early neonatal infections as well as low birth weight negatively influenced the success of INSURE-therapy.

\section{P0-0766 EXPLORING A PHYSIOLOGICAL DEFINITION FOR BRONCHOPULMONARY DYSPLASIA}

${ }^{1} \mathrm{~A}$ Kavanagh, ${ }^{1} \mathrm{~A}$ Hollitt, ${ }^{1} \mathrm{E}$ Skuza, ${ }^{1} \mathrm{P}$ Berger, ${ }^{2} \mathrm{JG}$ Jones, ${ }^{3} \mathrm{GG}$ Lockwood, ${ }^{4} \mathrm{~K}$ Tan. ${ }^{1}$ The Ritchie Centre, Monash Institute of Medical Research, Melbourne, Australia; ${ }^{2}$ University Department of Anaesthesia, Addenbrookes Hospital, Cambridge, UK; ${ }^{3}$ Anaesthetic Department, Hammersmith Hospital, London, UK; ${ }^{4}$ Monash Newborn, Monash Medical Centre, Melbourne, Australia

\subsection{6/archdischild-2014-307384.1405}

Background and aims Current definitions for bronchopulmonary dysplasia (BPD) lack objectivity. A physiological definition for BPD where the level of shunt and the reduction in ventilation-perfusion ratio serve as an objective grading of severity has been suggested. Shunt and reduced VA:Q can be measured noninvasively by determining the relationship of arterial oxygen saturations $(\mathrm{SpO} 2)$ to the fraction of inspired oxygen (FiO2). Our aims were to: 1 . quantify shunt and reduced VA:Q in infants with BPD and in preterm infants without BPD. 2.correlate shunt and VA:Q to clinical grading of severity where possible

Methods The group study population consisted of 10 infants (two with 'No BPD', two with 'Mild BPD' and six with 'Severe BPD') based on the NIH grades of BPD severity. Stepwise alterations in $\mathrm{FiO} 2$ were made, whilst ensuring infants stayed within the Monash Newborn SpO2 alarm limits. A two compartmental model of gas exchange was used to derive the $\mathrm{SpO} 2$ vs. FiO2 curves and values for shunt and VA:Q.

Results Five out of six infants with 'Severe BPD' and one infant with 'Mild BPD' had VA:Q well below normal, range 0.34 to 0.56 . Two infants with 'No BPD' and two infants with BPD, had $\mathrm{SpO} 2$ vs. FiO2 curves suggesting no impairment in gas exchange. The level of shunt and reduction in VA:Q did not consistently reflect the clinical grading of BPD.

Conclusions Our results reinforce the need for a more objective definition of BPD as the possibility of misclassification using the clinical definition occurred on three occasions.

\section{PO-0767 OVERNIGHT PULSE OXIMETRY STUDIES FOR PRETERM INFANTS WITH CHRONIC NEONATAL LUNG DISEASE - AN AUDIT FROM A LEVEL III NICU}

${ }^{1} \mathrm{~A}$ Resdiani, ${ }^{2} \mathrm{M}$ Ibrahim, ${ }^{3} \mathrm{P}$ Berger, ${ }^{2} \underline{\mathrm{K}}$ Tan. ${ }^{1}$ Department of Paediatrics, Monash University, Melbourne, Australia; ${ }^{2}$ Monash Newborn, Monash Medical Centre, Melbourne, Australia; ${ }^{3}$ The Ritchie Centre, Monash Institute of Medical Research, Melbourne, Australia

10.1136/archdischild-2014-307384.1406
Background and aims Infants with chronic neonatal lung disease (CNLD) often require home oxygen therapy. Overnight pulse oximetry monitoring (12-18 h duration) is useful for confirming need for oxygen therapy. We aimed to audit practise in our NICU after implementation of changes in our unit's overnight oximetry monitoring policy (Masimo Rad 7 pulse oximeters, PROFOX analysis software and new clinical protocol).

Methods We conducted a retrospective review of overnight oximetry from two full year (Jan 2012-Dec 2013). Clinical data were abstracted from medical records and archived oximetry reports generated from PROFOX were also retrieved.

Results 57 infants with CNLD had overnight oximetry performed in our centre with about two studies each.

\begin{tabular}{llll} 
Abstract PO-0767 Table 1 & & & \\
\hline & 2012 & 2013 & p-value \\
Infants with CNLD & 27 & 30 & \\
Number of studies & 61 & 64 & \\
Median Gestational Age (days) & $27(23-32)$ & $26.5(23-30)$ & \\
Median Postnatal Age Studied (days) & $110(14-326)$ & $104.5(20-822)$ & \\
Median time from discharge of oximetry (days) & $8.1 \pm 7.5$ & $7.9 \pm 5.3$ & 0.88 \\
Median oxygen flow-rate (L/min) & $0.125(0-0.5)$ & $0.05(0-0.5)$ & 0.41 \\
No of studies changing oxygen therapy (\%) & $19(31.1)$ & $29(45.3)$ & 0.19 \\
Infants -home oxygen therapy (\%) & $16(59.3)$ & $22(73.3)$ & 0.19 \\
Median Recording Time (Hr) & $12.3(0.08-25.5)$ & $12.5(7.4-111.5)$ & 0.12 \\
Desaturation Events $>3$ min & $6(0-19)$ & $7(0-50)$ & \\
Desaturation Events $<3$ min & $212(25-1102)$ & $313.5(28-1605)$ & \\
\hline
\end{tabular}

Conclusions Overnight oximetry studies were performed just over 7 days from discharge; with the PROFOX reports increasingly affecting a change in oxygen therapy (flow rate delivered). These infants also experienced numerous brief oxygen desaturations. There was an increased trend of infants discharged home with oxygen.

\section{PO-0768 EVALUATION OF VENTILATORY PARAMETERS, SHORT AND LONG TERM MORBIDITIES IN PRETERMS VENTILATED WITH EITHER PSV+VG OR SIMV+VG}

S Unal, E Ergenekon, S Aktas, N Altuntas, S Beken, E Kazanci, F Kulali, IM Hirfanoglu, E Onal, C Turkyilmaz, E Koc, Y Atalay. Department of Pediatrics Division of Neonatology, Gazi University Faculty of Medicine, Ankara, Turkey

\subsection{6/archdischild-2014-307384.1407}

Introduction Volume guarantee (VG) ventilation is frequently used for newborns, mostly combined with SIMV or $\mathrm{A} / \mathrm{C}$ modes. Aim of this study was to compare effect of SIMV+VG or PSV $+\mathrm{VG}$ ventilation on ventilatory and laboratory parameters and clinical findings.

Patients and methods Preterms with RDS $<34$ thgestational age (GA)requiring mechanical ventilation in the first $12 \mathrm{~h}$ were randomised to either SIMV+VG or PSV+VG after surfactant treatment. Patients were ventilated with Draeger Babylog 8000+. Set and measured ventilatory parameters were downloaded by Babyview ${ }^{\circledR}$ software for $72 \mathrm{~h}$ unless extubation or need for HFO ventilation occurred. Actual peak inspiratory pressure (PIP), set and measured tidal volume (TV), mean airway pressure (MAP) and $\mathrm{FiO}_{2}$ were analysed. If measured TV percentage was between $80-120 \%$ of set TV, it was considered appropriate. 


\section{Abstract P0-0770 Table 1}

\begin{tabular}{|c|c|c|c|c|c|}
\hline & & Vte $(\mathrm{mL} / \mathrm{kg})$ & $\mathrm{CO}_{2}(\mathrm{mmHg})$ & RC change per breath/inflation (AU/kg) & $A B$ change per breath/inflation ( $A U / \mathrm{kg})$ \\
\hline \multirow[t]{2}{*}{ SI (median (IQR)) } & & $5.1(1.0-10.4)$ & $10(2-19)$ & $6(-73-114)$ & $47(1-146)$ \\
\hline & Inflations only & $4.2(2.3-8.7)$ & $2(2-6)$ & $6(-15-41)$ & $46(19-100)$ \\
\hline PPV (median (IQR)) & Inflations coinciding with breathing & $6.9(5.2-9.8)$ & $18(12-24)$ & $4(-41-45)$ & $24(-9-102)$ \\
\hline \multicolumn{2}{|c|}{ Breathing (median (IQR)) } & $5.7(2.4-8.9)$ & $20(9-32)$ & $-7(-57-38)$ & $97(23-221)$ \\
\hline \multicolumn{2}{|l|}{$\mathrm{p}$-value } & ns & $<0.0001$ & 0.003 & $<0.0001$ \\
\hline
\end{tabular}

Results 42 patients (21 PSV+VG, 21 SIMV+VG) were enrolled. Median GA were 29 weeks and BW were 980,0 and 870,0 gr in each group. Demographic characteristics were similar. 'Appropriate TV' was higher in PSV+VG group. PIP, MAP and $\mathrm{FiO}_{2}$ were similar in two groups. Hypocarbia, hypercarbia, hyperoxemia and hypoxemia incidences were not different. PSV $+\mathrm{VG}$ group were less tachycardic than SIMV+VG group. Acute and chronic prematurity problems including chronic lung disease (CLD) defined as oxygen requirement at 36th GA were not different.

Conclusion PSV+VG was associated with higher 'appropriate TV' without any adverse effects and similar CLD occurence. These findings can support the beneficial use of PSV+VG which is more physiologic due to better inspiratory - expiratory synchrony.

\section{PO-0769 THE PRETERM PIG AS A MODEL FOR ACUTE LUNG DISEASE}

${ }^{1} \mathrm{M}$ van der Merwe, ${ }^{2} \mathrm{AF}$ Caminita, ${ }^{1} \mathrm{~B}$ Hance, ${ }^{1} \mathrm{KK}$ Buddington, ${ }^{1}$ RK Buddington. ${ }^{1}$ Health and Sport Sciences, University of Memphis, Memphis, USA; ${ }^{2}$ Draeger Medical Inc, Draeger Medical Inc, Telford, USA

\subsection{6/archdischild-2014-307384.1408}

Background and aims Despite advances in ventilation support, acute lung disease (ALD) remains the leading cause of morbidity, mortality, and disability after preterm birth. There is a need for a spontaneous translational model of ALD after preterm birth.

Methods Preterm pigs delivered at gestation days (GD) 98, 100, 102 , and 104 days were provided ventilation support using supplemental oxygen (NC), bubble Continuous Positive Airway Pressure (bCPAP; 7-8 cm $\mathrm{H}_{2} \mathrm{O}$ ), or mechanical ventilation (MV; Pressure Control Ventilation with Volume Guarantee; $5 \mathrm{ml} / \mathrm{kg}$; PEEP $5 \mathrm{~cm} \mathrm{H}_{2} \mathrm{O}$ ). Monitoring included pulse oximetry, arterial blood gases, and radiography. Lungs were harvested after $24 \mathrm{~h}$ or after premature death for histology and measurements of surfactant protein B, phosphatidylcholine, and cytokines.

Results All pigs breathed spontaneously. Lungs at GD 98 and 100 were consolidated with immature alveolar architecture, minimal surfactant protein B expression, and MV was essential for 24 h survival. GD 102 pigs had alveoli lined by pneumocytes and surfactant was released in response to MV. Blood gases and radiography for NC and bCPAP pigs 1-2 h after delivery revealed limited recruitment and mortality at $24 \mathrm{~h}$ was $66 \%$ (35/ 53 ) and 69\% (9/13), respectively. GD 104 pigs had higher densities of thin walled alveoli that secreted surfactant and MV was not essential.

Conclusions Preterm pigs have developmental changes in ventilation inadequacies that mimic those of preterm infants and represent a spontaneous model of ALD that is clinically relevant, compatible with standards of chronic neonatal intensive care, and is an alternative for nonhuman primates and lambs.

\section{P0-0770 RESPIRATORY INDUCTANCE PLETHYSMOGRAPHY AND EXPIRED CO2 LEVELS OF PRETERM INFANTS AT BIRTH}

${ }^{1} \mathrm{~J}$ van Vonderen, ${ }^{2} \mathrm{G}$ Lista, ${ }^{2} \mathrm{~F}$ Cavigioli, ${ }^{3} \mathrm{SB}$ Hooper, ${ }^{1} \mathrm{AB}$ te Pas. ${ }^{1}$ Neonatology, Leiden University Medical Center, Leiden, Netherlands; ${ }^{2}$ Neonatology, v. Buzzi Children's Hospital ICP, Milan, Italy; ${ }^{3}$ The Ritchie Center, Monash Institute of Medical Research, Melbourne, Australia

\subsection{6/archdischild-2014-307384.1409}

Background Preterm newborns often need respiratory support for lung liquid clearance and aeration. Previous studies provided tidal volumes during positive pressure ventilation (PPV) and breathing, but very little is known how efficient these are in lung recruitment and gas exchange. Aim was to measure tidal volume, functional residual capacity (FRC) changes and gas exchange during respiratory support in preterm infants at birth.

Methods In preterm newborns needing respiratory support the following measurements were performed: 1) expired tidal volumes (Vte $(\mathrm{mL} / \mathrm{kg})$ ) using respiratory function monitoring, 2) changes in FRC $(\mathrm{AU} / \mathrm{kg})$ per breath using Respiratory Inductance Plethysmography (bands placed around the rib cage (RC) and abdomen (AB)), 3) expired $\mathrm{CO}_{2}$ using a volumetric $\mathrm{CO}_{2}$ monitor. For respiratory support a T-piece resuscitator and mask were used with PIP $25 \mathrm{~cm} \mathrm{H}_{2} \mathrm{O}$ and PEEP $5 \mathrm{~cm} \mathrm{H}_{2} \mathrm{O}$. Data was analysed during sustained inflation (SI), the first 30 s of PPV and breathing on CPAP.

Results 15 infants were included (median (IQR) gestational age 28 (27-31) weeks, birth weight 1080 (994-1300) grams). There was no difference in Vte between SI, PPV and breathing (table). Gas exchange was more efficient during breathing and inflations coinciding with breathing compared to SI and inflations only (table). Little change occurred during the SI, PPV and breathing measured at the RC. In contrast, there was FRC gain at the $\mathrm{AB}$ during the SI, PPV and most with breathing.

Conclusions While tidal volumes during PPV and breathing were similar, breathing was more effective in gas exchange and caused more gain in FRC than PPV.

\section{PO-0771 THE EFFECT OF EXOGENOUS SURFACTANT THERAPY ON LUNG MECHANICS IN VERY PRETERM INFANTS}

F Yavanoglu Atay, O Bozkurt, G Kutman, FE Canpolat, SUNA Oguz, N Uras, U Dilmen. Neonatology, Zekai Tahir Burak Women Health Care Education and Research Hospital, Ankara, Turkey

\subsection{6/archdischild-2014-307384.1410}

Introduction and aim Surfactant replacement is a corner stone therapy for respiratory distress syndrome (RDS) and has been shown to be both safe and efficacious for premature infants. The aim of this study was to assess the immediate changes in lung mechanics caused by administration of two different natural surfactants. Secondary aim of this study was to determine the 